\title{
PERCEPÇÃO DE ACADÊMICOS DE ENFERMAGEM SOBRE O CUIDAR DE IDOSOS
}

Fabíola de Araújo Leite Medeiros ${ }^{1}$, Daísy Vieira de Araújo², Larissa Nogueira de Siqueira Barbosa ${ }^{3}$

RESUMO: Estudo analítico e descritivo cujo objetivo foi descrever e analisar a percepção dos acadêmicos de enfermagem sobre a formação em Gerontologia. A amostra foi composta por 57 acadêmicos de enfermagem da Universidade Estadual da Paraíba. Diante da análise de conteúdo foram selecionadas duas categorias temáticas: os elementos envolvidos no processo de cuidar de idosos (incluindo a exigência da subjetividade e conhecimento especializado, além do reconhecimento social ao idoso); e outra esteve relacionada com práticas e saberes do cuidar de idosos necessários ao ensino de enfermagem. Sugere-se que mais estudos se direcionem na reflexão dos currículos e em discussões sobre a saúde do idoso visando à formação de um enfermeiro apto para enfrentar os desafios do envelhecimento da população.

PALAVRAS-CHAVE: Idoso; Enfermagem; Educação.

\section{PERCEPTION OF ACADEMIC OF NURSING ON CARE FOR AGED}

ABSTRACT: This article refers to a descriptive and analytical study whose main objective was to describe and analyze the perceptions of nursing academics about education in Gerontology. The sample was composed by 57 nursing academics of Paraiba State University. Given the content analysis, two theme categories were selected: the elements involved in caring for elderly (including the requirement of subjectivity and expertise, in addition to the social recognition to the elderly); and another was related to practices and knowledge needed to care for the elderly in nursing education. It is suggested that more studies should be directed in order to the reflection on the nursing schools' curriculum, and discussions about the elderly health should be carried out in order to enable the educate nurses that are able to meet the challenges of an aging population.

KEYWORDS: Aged; Nursing; Education.

\section{PERCEPCIÓN DE ACADÉMICOS DE ENFERMERÍA SOBRE EL CUIDADO DE ANCIANOS}

RESUMEN: Estudio analítico y descriptivo cuyo objetivo fue describir y analizar la percepción de los académicos de enfermería sobre la formación en Gerontología. La muestra estaba compuesta por 57 académicos de enfermería de la Universidad Estadual de Paraíba. Teniendo en cuenta el análisis del contenido fueron seleccionadas dos categorías temáticas: los elementos envueltos en el proceso del cuidado de las personas de edad avanzada (incluyendo la exigencia de la subjetividad y el conocimiento especializado, además del reconocimiento social del anciano); y otra estuvo relacionada con las prácticas y los conocimientos sobre el cuidado de ancianos necesarios para la enseñanza de enfermería. Se sugiere que más estudios sean direccionados hacia la reflexión de los currículos y en discusiones sobre la salud del anciano visando a la formación de un enfermero apto para enfrentar los retos del envejecimiento de la población.

PALABRAS CLAVE: Anciano; Enfermería; Educación.

${ }^{1}$ Enfermeira. Mestre. Professora. Departamento de Enfermagem. Universidade Estadual da Paraíba-UEPB. Orientadora. ${ }^{2}$ Enfermeira. Mestre. Professora. Departamento de Enfermagem da Universidade Estadual da Paraíba-UEPB. Co-orientadora. ${ }^{3}$ Enfermeira. Centro de Apoio Psicossocial-CAPS-PB.

Autor correspondente:

Fabíola de Araújo Leite Medeiros

Rua José do Patrocínio, 330 - 58107-603 - Campina Grande-PB.

Recebido: 24/09/08

E-mail: eduardormedeiros@yahoo.com.br. 


\section{INTRODUÇÃO}

Ainda que seja um país jovem, o Brasil vem demonstrando uma transição em seu perfil populacional, prevendo-se que no ano de 2025, ocupará o sexto lugar entre os países com maior quantitativo de idosos, qual sejam pessoas com mais de sessenta anos de idade. Dentro desse cenário atual, torna-se necessário refletir a formação de profissionais capacitados para enfrentar a mudança e a demanda de serviços devido às alterações epidemiológicas vigentes com o aumento da longevidade.

É reconhecida na atualidade que está havendo um aumento na produção científica sobre o envelhecimento. A transição demográfica atual repercute outro fenômeno chamado de transição epidemiológica relacionado com alterações no perfil da morbidade e as causas de morte dessa população. $\mathrm{O}$ que antes era preocupação dos profissionais de saúde, o caso das doenças transmissíveis, hoje há uma necessidade de se investir mais nas doenças crônicodegenerativas, patologias mais comuns quando a população envelhece ${ }^{(1-2)}$.

A Enfermagem, como profissão de saúde, é reconhecida tradicionalmente como importante componente individual de serviços necessários ao atendimento do idoso doente como também para atenção ao idoso sadio, visando ajudá-lo a manter independência e apoiá-lo no autocuidado, a fim de garantir uma melhor qualidade de vida ${ }^{(3)}$.

O cuidar de idosos requer o conhecimento de uma assistência gerontológica complexa, tendo como pré-requesitos para esse cuidado a busca da história de vida, as prioridades de cada um, as lições do corpo e seus significados individuais, a capacidade funcional de manter as atividades de vida diária, além de outros problemas envolvidos com o processo de envelhecimento $^{(4)}$.

Levando em consideração todas as modificações físicas, psíquicas e sociais que se alteram no processo de envelhecimento humano, o cuidado de Enfermagem necessita ir além da técnica, da quantificação e da observação de sinais e sintomas. Somente o enfermeiro crítico, formado através de uma metodologia problematizadora, será capaz de propor e executar mudanças nos modelos do cuidar, e mais especificamente, quando este cuidar se refere a um grupo específico, como é o caso dos idosos. Certamente, para que este profissional exista, deverão ser incluídas na sua formação, como acadêmico, abordagens acerca do desenvolvimento da consciência profissional, através da valorização do ser humano e de seu potencial para se cuidar, cuidar e ser cuidado(5-6).

Conforme apontam as Diretrizes Curriculares Nacionais do Curso de Graduação em Enfermagem, materializada pela Resolução CNE/CES n ${ }^{\circ}$. 3, de 7 de novembro de 2001, em seu artigo $5^{\circ}$, o enfermeiro deve ser dotado de competências e habilidades para atuar nos diversos programas de assistência à saúde, dentre eles o programa de atenção integral ao idoso ${ }^{(7)}$, sendo a graduação, portanto, um espaço privilegiado para formação de profissionais capazes de atender às necessidades dos serviços de saúde, em termos de força de trabalho, na perspectiva da valorização das necessidades sociais de saúde ${ }^{(8)}$.

Torna-se essencial afirmar que a Enfermagem na assistência ao idoso trabalha na rede formal de apoio (hospitais, clínicas, asilos) na relação interpessoal profissional-ser cuidado, como também trabalha a rede informal de apoio ao idoso (destinada aos cuidados domiciliares de familiares, de organizações não governamentais, de cooperativas solidárias, vizinhos) ${ }^{(9)}$. Em ambas as redes assistenciais, os enfermeiros exercem funções direcionadas à atividade junto ao idoso, família e comunidade ${ }^{(7,9)}$.

Como prática social, a Enfermagem inserida na produção de cuidados à saúde inscreve-se em um contexto no qual se articulam o sistema político, as organizações de produção de serviços de saúde, como mercados de absorção e demanda, e o setor de educação/formação profissional. A formação do enfermeiro não pode ser pensada de forma linear e pontual ${ }^{(8,10)}$. Nesse sentido, os Ministérios da Educação e da Saúde vêm apoiando as universidades na superação de suas contradições, a fim de habilitá-las a produzir profissionais dotados de competências política, ética, técnica e científica, comprometidos com as necessidades impostas pelo processo de Reforma Sanitária ${ }^{(8)}$.

Assim, criar um elo da situação demográfica e epidemiológica atual com a formação do profissional de Enfermagem compreende não só uma exigência de um novo panorama do mercado de trabalho, mas sim uma realidade social que influenciará num pensar e agir em relação ao cuidar específico à uma clientela especial. Surge, pois, uma indagação investigativa: qual a percepção dos acadêmicos de enfermagem sobre a formação em Gerontologia?

Para responder tal questionamento formulamos o seguinte objetivo: descrever e analisar a percepção 
dos acadêmicos sobre a formação em Gerontologia, tecendo uma reflexão sobre os saberes e as práticas necessárias dentro da atual situação demográfica.

\section{RECURSOS METODOLÓGICOS}

Trata-se de uma pesquisa do tipo exploratória com abordagem qualitativa. Foi realizada com 57 acadêmicos de enfermagem da Universidade Estadual da Paraíba-UEPB, situada no município de Campina Grande/PB, matriculados nas últimas séries da graduação ( $4^{\circ}$ e $5^{\circ}$ anos). Essa população se referia a aproximadamente $47 \%$ dos acadêmicos matriculados nos últimos anos da graduação. A opção por concluintes se deu pelo fato destes alunos já terem cursado mais de $50 \%$ das disciplinas do curso tendo maior possibilidade de ter tido contato com idosos durante o ensino clínico e estágios curriculares, o que ajudaria na resolução dos objetivos traçados pelo estudo. $\mathrm{O}$ período de realização da coleta de dados se deu nos meses de julho a setembro de 2007.

O instrumento para coleta de dados foi um formulário que contemplava perguntas semiestruturadas abordando perguntas do tipo: Em sua opinião, o que é cuidar de idosos? Qual a importância do cuidado de idosos dentro do curso de enfermagem? Você acha importante ter um componente na graduação que aborde exclusivamente a Saúde do Idoso? Qual a importância que a graduação de Enfermagem tem à Gerontologia? Quais sugestões você daria em relação ao cuidar de idosos no curso de graduação em Enfermagem?

Os dados foram analisados e categorizados seguindo a análise do conteúdo ${ }^{(12)}$. As falas abertas foram submetidas à análise temática, sendo classificadas a partir de uma pré-análise (organizando o material coletado e sistematizando as idéias através de leitura meticulosa das respostas obtidas pelo questionário). Depois foi feito uma categorização e quantificação das unidades de registro de acordo com as falas escritas ${ }^{(12)}$.

Este estudo foi desenvolvido levando em consideração os aspectos éticos de pesquisas envolvendo seres humanos, preconizado na Resolução 196/96 do Conselho Nacional de Saúde ${ }^{(11)}$. O projeto de pesquisa foi aprovado sob protocolo de ${ }^{\circ}$ 0155.0.133.000 pelo Comitê de Ética em Pesquisa da UEPB e os critérios de inclusão na pesquisa foram à voluntariedade em participar com autorização registrada em um termo de consentimento livre e esclarecido.

\section{RESULTADOS E DISCUSSÃO}

Dos acadêmicos que participaram deste estudo, 44 (77\%) pertencem ao sexo feminino e 13 (23\%) ao sexo masculino. Percebe-se que vem ocorrendo um aumento da freqüência do gênero masculino no curso de Enfermagem, fenômeno este, que vem substituindo a imagem social de que o curso de Enfermagem seria mais voltado ao público feminino. Importante elucidar que Enfermagem se refere a uma profissão baseada na arte e ciência do cuidar. E o cuidar é um ato inerente ao ser humano. Todo ser humano adulto tem a capacidade de cuidar de outro ${ }^{(3)}$. A faixa etária dos participantes compreendia idades entre 20 a 25 anos.

Da população estudada, 28 (49\%) acadêmicos pertenciam ao $4^{\circ}$ ano e 29 (51\%) ao último ano do curso de Enfermagem, tendo já cursado disciplinas da área profissional.

Foram questionados aos acadêmicos quais os componentes teóricos do curso de Enfermagem da UEPB que contemplam o cuidar de idosos. Eles responderam que as disciplinas de Enfermagem em Clínica Médica, Enfermagem em Clínica Cirúrgica, UTI, Saúde Coletiva, Patologia, Semiotécnica e Semiologia da Enfermagem, Urgência e Emergência, Humanização da Saúde e Metodologia da Assistência de Enfermagem, todas estas citam algum conteúdo com abordagem sobre o cuidar de idosos.

Foi questionado também se nos estágios práticos das disciplinas do curso, há menção da atenção gerontológica, como atuação de enfermagem a cuidados específicos para a faixa etária acima dos 60 anos de idade. Sendo assim, 52,6\% dos acadêmicos afirmam que em algumas disciplinas práticas sim, porém 43,7\% citaram que não há uma abordagem direcionada aos aspectos gerontológicos na atuação prática do cuidar em enfermagem.

Destaca-se que 89,4\% dos acadêmicos referem já terem tido alguma vivência com o paciente idoso, embora a maioria dos acadêmicos pesquisados (61\%) referirem que o curso de enfermagem da UEPB tem dado pouca importância teórica aos assuntos da Gerontologia.

Diante dos dados supracitados, há uma necessidade de reflexão acerca dos currículos de enfermagem e da sua atuação na prática do ensino, pesquisa e extensão para que haja inserção dos conteúdos da Gerontologia e Geriatria nos conteúdos programáticos. A composição populacional modificada e em conjunto as patologias que devem ser 
supervisionadas, controladas e prevenidas para essa faixa etária exigem um profissional de saúde que saiba lidar com as nuances específicas dessa faixa etária ${ }^{(13)}$.

Os profissionais de saúde, em especial os enfermeiros, devem abordar o idoso considerando as especificidades decorrentes do envelhecimento. É essencial que na graduação haja discussões sobre a Gerontologia no sentido de não só preparar o acadêmico para a nova composição demográfica e epidemiológica, como também de modificar a visão estereotipada, exclusa e preconceituosa, muitas vezes, imposta pela sociedade em relação ao processo de envelhecimento $^{(9)}$.

Dentro desse contexto e de acordo com as falas encontradas foi possível direcionar este estudo sob duas categorias temáticas: Categoria I - Elementos envolvidos no processo de cuidar de idosos; e Categoria II - Saberes e práticas do cuidar de idosos necessários ao ensino de enfermagem. Dentre essas categorias foram determinadas subcategorias expostas no Quadro 1.

Quadro 1 - Distribuição das categorias e subcategorias

\begin{tabular}{|l|}
\hline Categoria I - Elementos envolvidos no processo de cuidar \\
de idosos \\
- Cuidar de idosos exige sensibilidade \\
- Cuidar de idosos exige conhecimento técnico \\
especializado \\
- Cuidar de idosos é um reconhecimento social \\
\hline Categoria II - Saberes e práticas do cuidar de idosos \\
necessários ao ensino de enfermagem \\
- Enfermagem como profissão de saúde integralizando os \\
cuidados às faixas etárias especiais \\
- Criação de um componente curricular em Gerontologia \\
- Inserção do tema Saúde do Idoso em todos os \\
componentes curriculares \\
- Criação de práticas de grupos de idosos e \\
docente/discente do curso.
\end{tabular}

\section{Categoria I: Elementos envolvidos no processo de cuidar de idosos}

\section{Cuidar de idosos exige sensibilidade}

Nessa categoria foram analisadas respostas que tinham como conteúdo expressões de algum sentimento ou subjetividade na atuação do cuidar de idosos. Houve uma freqüência de respostas, cerca de 45\% dos acadêmicos relacionaram cuidar de idosos com necessidade de humanização e sensibilização por parte de quem cuida:

O cuidar de idoso é muito além que simples procedimentos feitos, se referem a criar vínculo de carinho, prestar assistência nas diversas áreas que a idade requer (S.13).

Cuidar de idosos é poder orientá-lo quanto aos possíveis problemas da terceira idade, amando-os e respeitando-os com dignidade e ajudando-os a vencer os preconceitos impostos pela sociedade (S.25).

O elo interpessoal entre o cuidador e o ser cuidado se caracteriza, nestas falas, como uma relação humanística, que se expressa no envolvimento da equipe com o indivíduo. Esta interação pode variar de uma relação superficial até áquela mais profunda na qual se estabelece vínculos numa ligação de intimidade e de reciprocidade. Assim, a qualidade do cuidado está diretamente relacionada a um contato mais próximo e a qualidade da relação, o que gera benefícios no processo terapêutico ${ }^{(10)}$.

Um sistema humanístico de valores sustenta a construção da ciência do cuidado. Dentro desse enfoque, trabalhar com o aprofundamento teórico dos paradigmas que envolvem o cuidar faz com que tecnologias mais subjetivas e mais completas sejam acrescidas a rotina terapêutica e curativa exigida para o enfermeiro que atua com todas as faixas etárias e em todos os ambientes terapêuticos, seja do indivíduo, família ou comunidade ${ }^{(4-13)}$. O acadêmico com essa percepção provavelmente está preparado para lidar com idosos, pois não exclui a subjetividade envolvida nas relações interpessoais do cuidado.

\section{Cuidar de idosos exige conhecimento técnico especializado}

Essa subcategoria revelou a necessidade que os acadêmicos de enfermagem têm em relação a cuidar especificamente do idoso. Aproximadamente, $40 \%$ das falas se dirigiam para tal necessidade:

É estabelecer as necessidades físicas e psíquicas do mesmo, analisando o contexto biopsicossocial em que está inserido para favorecer a qualidade de vida, exigindo maior conhecimento técnicocientífico (S.11.)

Observa-se pelos discursos dos acadêmicos que 
há uma necessidade de aperfeiçoamento específico para lidar com o idoso tendo em vista suas necessidades físicas, sociais e psíquicas que extrapolam o modelo biomédico, outrora agindo sobre a formação.

Sabe-se que o ambiente do cuidado dito como humanístico é aquele que providencia o desenvolvimento potencial do cuidador com o ser cuidado e vice-versa. Para que o elo interpessoal ocorra de maneira satisfatória no processo de cuidar, deve-se levar em consideração os aspectos tecnológicos e terapêuticos, deve haver aparato científico e perspicácia do enfermeiro com o ser humano que recebe o cuidado ${ }^{(13)}$.

\section{Cuidar de idosos é um reconhecimento social}

Para 15\% dos acadêmicos entrevistados, cuidar de idosos está relacionado a um processo de agradecimento social, fazer por eles, pois eles já fizeram muito pela sociedade quando jovens, e agora necessitando de cuidados, nada mais justo do que cuidar como retribuição social a que fora outrora construída:

O cuidar de idosos é um ato de reconhecimento por aquela pessoa que sempre cuidou de nós (S.55).

É uma gratificação por tudo aquilo que o idoso fez ao longo do tempo, que nessa gratidão demonstramos o quanto ele é importante (S.23).

Observa-se nesses discursos uma imagem mítica e esteriotipada do idoso associado ao velho. Surge, diante dessa categoria, uma necessidade de se refletir como o acadêmico de enfermagem tem consciência de sua atuação frente às necessidades do idoso.

Categoria II: Saberes e práticas do cuidar de idosos necessários ao ensino de enfermagem

Enfermagem como profissão de saúde integralizando os cuidados às faixas etárias especiais

A Enfermagem deve estar preparada para lidar com as necessidades dessa faixa etária. Mediante essa citação, 55\% dos participantes citaram em suas falas que direcionasse o cuidado de enfermagem a idosos como cuidados especiais relacionados às necessidades vivenciadas por indivíduos pertencentes à faixa etária acima dos 65 anos.
Como em qualquer fase da vida, a velhice também necessita de cuidados, e a enfermagem em sua assistência integral deve sistematizar os cuidados aos idosos (S.39).

É de fundamental importância já que nos deparamos no dia-a-dia com as situações em que o cuidado de enfermagem ao idoso muitas vezes se torna como total dependência para prolongar vida de forma a promover conforto e bem-estar (S.53).

Ao avaliar uma pessoa idosa, o enfermeiro deve ter em mente três aspectos: a perda que ocorre com a idade, o quadro patológico que se pode instalar e a existência de um componente genético-fisiológico inicial que dá uma característica própria a cada idade ${ }^{(16)}$.

\section{Criação de um componente curricular em gerontologia}

As falas a seguir emitem respostas que conferem à categoria a importância de se ter um conteúdo direcionado ao cuidar de idosos e a Enfermagem:

Em minha opinião deveria ter uma disciplina específica sobre a saúde dos idosos (S.36).

É importante a criação de um componente curricular de geriatria, pois as expectativas e a realidade mostram que a população de idosos está cada vez mais crescendo e com ela uma série de abordagens como as patologias crônicas e a qualidade dessa população idosa (S.44).

Deveria haver um enfoque maior em relação ao cuidar de idosos, inclusive com a organização de uma disciplina específica dedicada à geriatria em que fossem discutidos os diversos aspectos do envelhecimento humano (S.42).

A escassez de conhecimento gerontogeriátrico dos profissionais da saúde, a ausência de sintonia da maioria das instituições de ensino superior brasileiras com o atual processo de transição demográfica e suas conseqüências médico-sociais, a falta de campos específicos para a prática, além da inexperiência do corpo docente, são algumas das limitações presentes nos cursos de graduação da área da saúde ${ }^{(13)}$.

Especificamente para o curso de Enfermagem, 
a Organização Panamericana da Saúde (OPAS) recomenda que não sejam oferecidos conteúdos gerontogeriátricos parciais integrados a outras disciplinas, para não correr o risco de diluir ou reduzilos ao longo da grade curricular, bem como que os conteúdos sobre o cuidado ao idoso sadio precedam os referentes ao cuidado ao idoso enfermo ou institucionalizado, permitindo ao estudante visualizar essa etapa da vida como um período em que o ser humano sofre limitações da idade, mas que também pode desfrutar de uma fase de bem-estar e desenvolvimento ${ }^{(16-18)}$.

\section{Inserção do tema saúde do idoso em todos os componentes curriculares}

Tendo em vista a Interdisciplinaridade como possibilidade de manifestar ou correlacionar diversos tipos de conhecimentos fragmentados, existe a necessidade de caracterizar a própria natureza das disciplinas, para, então, sugerir uma ligação coerente dos conhecimentos que elas produzem.

Incluir o cuidado e atenção ao idoso igualmente a todos os clientes, em todos os componentes da graduação possíveis de relacionar (S.57).

Acredito que as disciplinas podem abordar essa temática de várias formas, sempre mostrando a assistência correta, pois pouco se sabe, tendo apenas a disciplina Enfermagem Clínica que aborda essa temática (S.34).

A noção mais conhecida de Interdisciplinaridade é a interação entre duas ou mais disciplinas para superar a fragmentação do conhecimento, implicando uma troca entre especialistas de vários campos de conhecimento, na discussão de um assunto, na resolução de um problema, com vistas à melhor compreensão da realidade ${ }^{(17)}$.

Criação de práticas de grupos de idosos e docente/ discente do curso

A importância de um trabalho além do ensino sobre cuidar de idosos, como o ensino superior reza por práticas que vão do ensino-aprendizagem, da pesquisa e da extensão, à ampliação dos horizontes de se voltar mais incentivos a práticas com grupos de idosos, isto seria de suma importância no processo de formação acadêmica. Dentro dessa perspectiva foram selecionadas as seguintes falas que direcionaram essa categorização específica:

Atividades com idosos fora dos bancos das salas de aula da universidade (S.9).

Deveriam ser criados grupos de idosos, com envolvimento nos projetos de extensão e pesquisa para quem se interessasse (S.46).

Entendendo-se que o ensino, pesquisa e extensão são elementos essenciais para consolidação das propostas pedagógicas das instituições de ensino superior, a criação de grupos de idosos com o intuito de ampliar o convívio, comunidade e universidade, se torna uma iniciativa para o (re)conhecimento de que a formação acadêmica deve refletir e atuar mais em fórmulas para uma maior inserção na sociedade científica, ou como meio de participação social.

\section{CONSIDERAÇÕES FINAIS}

Evidenciam-se através desse estudo que a sensibilidade, o envolvimento interpessoal e o conhecimento técnico-científico foram citados pelos os acadêmicos de enfermagem como peças básicas que devem ser utilizadas no ato de cuidar de idosos.

A percepção sobre a formação acadêmica em relação ao ato de cuidar de idosos sugere que deve haver um maior aprofundamento dos profissionais de saúde desde sua formação até o processo de atuação para que seja garantida uma assistência humanizada e de qualidade oferecida à faixa etária considerada como idosa. Outra prerrogativa a ser citada na percepção dos acadêmicos se refere à necessidade de capacitação profissional devido à demanda de serviços na área do cuidar de idosos. Fatos estes, que estimulam a preocupação do acadêmico em relação a sua formação desde a academia.

Sugere-se que mais estudos se direcionem na reflexão dos currículos previstos para a atualidade, preparando profissionais com visão crítica-reflexiva para construção de uma realidade na qual haja melhoria das condições de saúde da população em geral, incluindo a saúde ao idoso. Em relação á prática da enfermagem, torna-se essencial que haja maiores discussões sobre a saúde do idoso nos projetos políticos pedagógicos com visão para a transição demográfica vigente, como também para formação de um 
enfermeiro apto para cuidar diante das nuances do processo de envelhecimento.

\section{REFERÊNCIAS}

1. Cattani RB, Girardon-Perlini NMO. Cuidar do idoso doente no domicílio na voz de cuidadores familiares. Rev Eletron Enferm [periódico na Internet]. 2004 [acesso em 2008 Ago 10] 6(2). Disponível: http:// www.fen.ufg.br/revista

2. Santos SR, Ide KCA. Enfermagem e o idoso: necessidades e possibilidades para a realização de educação em serviço. Nursing. 2006; 103(9):1152-7.

3. Saldanha AL, Caldas CP. A saúde do idoso: a arte de cuidar. $2^{\mathrm{a}}$ ed. Rio de Janeiro: Interciência; 2004.

4. Figueiredo NMA, Tonini T. Gerontologia: atuação da enfermagem no processo de envelhecimento. São Paulo: Yendis; 2006.

5. Rizzotto MLF. História da Enfermagem e sua relação com a saúde pública. Goiânia: AB; 1999.

6. Sampaio SF. Qualidade de ensino de um curso de enfermagem: subsídio para construção de um modelo de avaliação. Texto Contexto Enferm. 1999; 8(1):273-85.

7. Brasil. Resolução da $\mathrm{CES} / \mathrm{CNE} n^{\circ} 3$, de 7 de novembro de 2001.Dispoe sobre as Diretrizes Curriculares Nacionais do Curso de Graduação em Enfermagem. Diário Oficial da União. Brasilia, 9 de out. 2001, seção 1. p. 37.

8. Araújo DV, Silva CCS, Silva, ATMC. Formação de força de trabalho em saúde: contribuição para a prática educativa em enfermagem. Cogitare Enferm. 2008 Jan/ Mar;13(1):10-7.

9. Néri AL, Pinto MEB, Sommenhalder C, Perracini, MR, Yuaso DR. Cuidar de idosos no contexto da família: questões psicológicas e sociais. $2^{\mathrm{a}}$ ed. Campinas: Alínea; 2006.

10. Sena RR, Christófaro MAC. A formação do enfermeiro. Caderno CE. 2002; 3(5):9-36.

11. Bardin L. Análise do conteúdo. Lisboa: Edição 70; 1997.

12. Brasil, Resolução nº 196 de 10 de outubro de 1996. Dispõe sobre as Diretrizes e Normas Regulamentadoras de Pesquisa Envolvendo Seres Humanos. Diário Oficial da União. Brasilia, 16 de out. 1996.

13. Montanholi LL, Tavares, DMS, Oiveira GRde, Simões
ALdeA. Ensino sobre idoso e gerontologia: visão do discente de enfermem no estado de Minas Gerais. Texto Contexto Enferm. 2006; 15(4):663-71.

14. Silva TJES. O Enfermeiro e a assistência a necessidade não física do cliente: o significado do fazer [tese]. Rio de Janeiro (RJ): Escola de Enfermagem Anna Nery. Universidade Federal do Rio de Janeiro; 1998.

15. Moreira WW. Qualidade de vida: Complexidade e educação. São Paulo: Papirus; 2001.

16. Stenveson JS, Gonçalves LHT, Alvarez AM. O cuidado e a especificidade da enfermagem geriátrica e gerontológica. Texto Contexto Enferm. 1997; 6(2):33-50.

17. Camacho ACLF. Agerontologia e a interdisciplinaridade: aspectos relevantes para a enfermagem. Rev LatinoAm Enferm. 2002; 10(2):229-33.

18. Weill PD’AU, Crema R. Rumo a nova transdisciplinaridade: sistemas abertos de conhecimento. $3^{\mathrm{a}}$ ed. São Paulo: Summus; 1993. 